\title{
28 Research Square \\ Soft X-ray Characterization of Halide Perovskite Film by Scanning Transmission X-ray Microscopy
}

Haeyeon Jun

LPICM, CNRS, Ecole Polytechnique, Institut Polytechnique de Paris

Hee Ryung Lee

LPICM, CNRS, Ecole Polytechnique, Institut Polytechnique de Paris

Denis Tondelier

LPICM, CNRS, Ecole Polytechnique, Institut Polytechnique de Paris

Bernard Geffroy

Université Paris-Saclay, CEA, CNRS, NIMBE, LICSEN

Philip Schulz

CNRS, École Polytechnique, IPVF, UMR 9006

Jean-Éric Bourée

LPICM, CNRS, Ecole Polytechnique, Institut Polytechnique de Paris

Yvan Bonnassieux

LPICM, CNRS, Ecole Polytechnique, Institut Polytechnique de Paris

Sufal Swaraj ( sufal.swaraj@synchrotron-soleil.fr)

Synchrotron SOLEIL

\section{Research Article}

Keywords: perovskite solar cells, STXM, x-ray microscopy, synchrotron, spectroscopy

Posted Date: February 25th, 2022

DOI: https://doi.org/10.21203/rs.3.rs-1395741/v1

License: (1) (i) This work is licensed under a Creative Commons Attribution 4.0 International License.

Read Full License

Version of Record: A version of this preprint was published at Scientific Reports on March 16th, 2022. See the published version at https://doi.org/10.1038/s41598-022-08256-3. 


\section{Abstract}

Organic-inorganic metal halide perovskites (MHPs) have recently been receiving a lot of attention due to their newfound application in optoelectronic devices, including perovskite solar cells (PSCs) which has reached power conversion efficiencies as high as $25.5 \%$. However, the fundamental mechanisms in PSCs, including the correlation of degradation with the excellent optoelectrical properties of the perovskite absorbers, are poorly understood. In this paper, we have explored synchrotron-based soft X-ray characterization as an effective technique for the compositional analysis of MHP thin films. Most synchrotron-based studies used for investigating MHP so far are based on hard X-rays (5-10 keV) which includes various absorption edges ( $\mathrm{Pb}$ L-edge, I L-edge, $\mathrm{Br} \mathrm{K}$-edge, etc.) but are not suited for the analysis of the organic component in MHPs. In order to be sensitive to a maximum number of elements, we have employed soft X-ray-based scanning transmission X-ray microscopy (STXM) as a spectro-microscopy technique for the characterization of MHPs. We examined its sensitivity to iodine and organic components, aging, or oxidation by-products in MHPs to make sure that our suggested method is suitable for studying MHPs. Furthermore, methylammonium triiodide with different deposition ratios of $\mathrm{Pbl}_{2}$ and $\mathrm{CH}_{3} \mathrm{NH}_{3} \mathrm{I}(\mathrm{MAl})$, and different thicknesses, were characterized for chemical inhomogeneity at the nanoscale by STXM. Through these measurements, we demonstrate that STXM is very sensitive to MHP chemical composition and homogeneity. Thus, we highlight the utility of STXM for an in-depth analysis of physical and chemical phenomena in PSCs.

\section{Introduction}

Organic-inorganic metal halide perovskites (MHPs) with $\mathrm{ABX}_{3}$ structure have been regarded as promising materials for various optoelectronic applications over the past few years. MHPs have remarkable optoelectronic properties, including a tunable bandgap, long charge carrier lifetimes, a small exciton binding energy, and a high absorption coefficient. ${ }^{[1-3]}$ These properties are well suited for electrical and optical applications such as photovoltaics, light-emitting diodes, resistive-switching memory devices, and field-effect transistors. ${ }^{[4-7]}$ Among various applications, perovskite-based solar cells (PSCs) conspicuously have achieved a certified power conversion efficiency (PCE) of $25.5 \%$ within ten years of development, comparable to that of monocrystalline silicon based solar cells. ${ }^{[8]}$

However, one of the challenging issues that hinders successful commercialization of PSCs is still low operational stability. The mechanisms of degradation in a bulk perovskite layer and interfaces between perovskite and transport layers are caused by various factors of the operational environment such as oxygen, moisture, heat, light, and electrical field. To improve the stability, many researchers have tried to investigate them by different techniques and have observed the degradation phenomena under various conditions. ${ }^{[9-11]}$ However, understanding of the intrinsic degradation attributed to light, heat, and electrical fields still remains vague due to the complexity of these materials. For a better understanding of the detailed mechanisms of the phenomena, advanced analytical techniques are needed, which enable investigation of nanoscale chemical composition with high sensitivity for every element. Combining 
spatially resolved chemical analysis with techniques probing material's local opotoelectronic properties is one way to understand the link between composition and performance of PSCs.

Since the advent of PSCs, characterizations based on synchrotron radiation have been employed to deepen our understanding of the correlation between non-stoichiometry and halide migration. Synchrotron radiation sources can produce highly collimated monochromatic X-rays with high flux. Owing to these beam characteristics, structural, chemical, and physical properties can be investigated across multiple length scales. ${ }^{[12]}$ In many instances, there have been applications of synchrotron radiation-based characterization, which has provided decisive answers to the physico-chemical mechanisms in MHPs that cannot be revealed by laboratory-based characterization. Adhyaksa et al. employed synchrotron-based nanoprobe X-ray diffraction (nano-XRD) to examine the effect of grainboundaries in MHP and verified that the disordered region at grain boundaries is amorphous giving rise to locally bright photoluminescence intensity, which is a quite anomalous grain boundary behavior. ${ }^{[13]} \mathrm{Li}$ et al. also used nano-XRD to study the influence of nanoscale residual strains on $\mathrm{CsPbBr}_{3}$ thin film on the

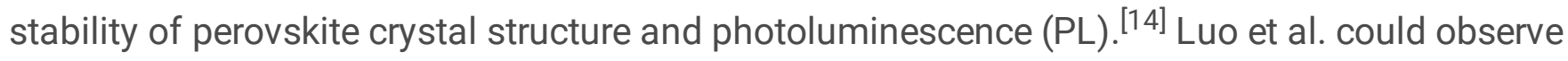
nanoscale heterogeneous distribution of chlorine/iodine mass ratio in $\operatorname{MAPb}\left(I_{1-x} \mathrm{Cl}_{x}\right)_{3}$ according to the method of synthesis and deposition using synchrotron-based nano X-ray fluorescence (XRF) microscopy. [15] With the same technique they observed in $\mathrm{CH}_{3} \mathrm{NH}_{3} \mathrm{PbBr}_{3}$ an increase of local bromine/lead atomic ratio under an applied electric field correlated with a local increase of photoluminescence. Furthermore, Stuckelberger et al. carried out nano-XRF with X-ray beam-induced current (XBIC) on mesoscopic $\mathrm{MAPb}_{3}$ solar cells. The combined XRF/XBIC measurements give valuable correlation between elemental distributions and charge collection efficiency. ${ }^{[16]}$ Philippe et al. demonstrated the depth-dependent distribution of $\mathrm{Cs}$ and $\mathrm{Rb}$ and the role of the distributions in mixed-cation RbCsMAFA perovskite with hard X-ray photoelectron spectroscopy (HAXPES). ${ }^{[17]}$ In addition, Sekimoto et al. performed HAXPES to investigate the effect of hole transport layer $(\mathrm{HTL})$ on light-induced degradation and demonstrated the degree of accumulation of iodine and lead at the interface between perovskite and contact layer according to HTL. ${ }^{[18]}$

The majority of these synchrotron-based studies of MHPs have employed hard X-rays in the range 5-10 $\mathrm{keV}$ primarily because the interesting X-ray absorption edges related to some of the elements ( $\mathrm{Pb}$ L-edge, I L-edge, $\mathrm{Br}$ K-edge, etc.) in MHP compounds lie in the hard X-ray regime. ${ }^{[17,19-21]}$ On the other hand, the soft X-ray regime does include the inner shell $\mathrm{X}$-ray absorption edges of carbon and nitrogen (C K-edge and $\mathrm{N} \mathrm{K}$-edge) and the outer shell X-ray absorption edges of $\mathrm{Pb}$ and I ( $\mathrm{Pb} \mathrm{N}$-edge and I M-edge) in the same regime contributing in the form of a strong background (Figure 1). While soft X-ray could be considered a complicated regime to investigate MHPs, it does provide the opportunity to investigate the organic component of MHPs in conjunction with the lead halide component.

In principle, this energy range could be used to investigate changes in the spectroscopic fine structure of the carbon K-edge at $284 \mathrm{eV}$ and the nitrogen K-edge at $405.5 \mathrm{eV}$, to explore the chemical changes at interfaces between perovskite active layers and charge transport layers ${ }^{[22]}$ as well as ion migration in the 
perovskite film itself. ${ }^{[23]}$ We would like to point out that the I $\mathrm{M}$ absorption edge as tabulated in the Henke database ${ }^{[24]}$ was found to be $20 \mathrm{eV}$ below $[619 \mathrm{eV}]$ that found in our studies [ $\left.640 \mathrm{eV}\right]$. As pointed out by Hansen ${ }^{[25]}$ and Comes et al. ${ }^{[26]}$, the tabulated values need further investigation. In order to overcome this discrepancy, we have blue-shifted the iodine M-edge tabulated value by $20 \mathrm{eV}$ in the range of [620-700 eV] before simulating the spectra using the software aXis2000 ${ }^{[27]}$. The simulation parameters used are mentioned in the Figure 1.

In this paper, we have used scanning transmission X-ray microscopy (STXM) measurements in the soft Xray regime (270-800 eV) to demonstrate the possibility of spectroscopy as well as microscopy using soft X-rays (soft X-ray spectro-microscopy). STXM is an experimental technique (see Figure 2 for schematics) based on X-ray absorption spectroscopy (XAS). In most of the STXM studies the "fine structure" near the absorption edge is specifically exploited. This is referred as near edge X-ray absorption fine structure (NEXAFS) spectroscopy or X-ray absorption fine structure (XAFS). In XAS, the absorption spectra are usually represented in terms of optical density (OD) given as ${ }^{\ln \left(\frac{I_{0}}{I}\right)}$ where ${ }^{I_{0}}$ is the incident photon flux and $/$ is transmitted photon flux. ${ }^{I}$ and $I_{o}$ (and hence OD) are correlated to the mass absorption coefficient $(\mu(E))$, density $(\rho)$;

and thickness (t) from from Beer-Lambert law as shown in Equation (1).

$$
\begin{aligned}
& I=I_{0} e^{(-\mu(E) \rho t)} \\
& \ln \left(\frac{I_{0}}{I}\right)=O D=\mu(E) \rho t
\end{aligned}
$$

This spectro-microscopy technique allows us to obtain quantitative chemical composition maps of the bulk of materials with a high spatial resolution ( $30 \mathrm{~nm}$ in this study).

In order to demonstrate the utility of soft X-ray STXM for the characterization of MHPs, we have verified its sensitivity to (a) various elements/components that are contained in MHPs and (b) various aging or oxidation by-products. To achieve the above objectives, the following samples were prepared: methylammonium lead iodide $\left(\mathrm{CH}_{3} \mathrm{NH}_{3} \mathrm{Pbl}_{3}, \mathrm{MAPbl}\right)$, lead iodide $\left(\mathrm{Pbl}_{2}\right)$, methylammonium iodide $\left(\mathrm{CH}_{3} \mathrm{NH}_{3} \mathrm{l}, \mathrm{MAl}\right)$, lead chloride $\left(\mathrm{PbCl}_{2}\right)$, potassium iodide $(\mathrm{KI})$, and lead oxide $(\mathrm{PbO})$. $\mathrm{Pbl}_{2}$ and $\mathrm{MAl}$ are major components of the $\mathrm{MHP}, \mathrm{MAPbl}_{3} . \mathrm{PbCl}_{2}$ and $\mathrm{KI}$ films are used as a comparison standard to test whether STXM is sensitive to $\mathrm{C}, \mathrm{N}, \mathrm{Pb}$ and I in this energy range. The $\mathrm{X}$-ray absorption spectrum of $\mathrm{PbO}$ obtained from STXM is measured to identify the by-product issued from the degradation of $\mathrm{MAPbl}_{3}(\mathrm{MHP}$ in this study). Furthermore, $\mathrm{MAPbl}_{3}$ samples with different $\mathrm{Pbl}_{2}: \mathrm{MAl}$ ratios and of various thicknesses were prepared and compared to highlight the chemical and thickness sensitivity of STXM.

\section{Results And Discussions}




\subsection{Identification of components and by-products}

Figure 3a shows NEXAFS spectra of $\mathrm{Pbl}_{2}, \mathrm{MAl}_{1} \mathrm{PbCl}_{2}$, and $\mathrm{Kl}$, covering the $\mathrm{C}$ K-edge, $\mathrm{N} \mathrm{K}$-edge, $\mathrm{O}$ K-edge, I $\mathrm{M}$-edge, and $\mathrm{Pb} \mathrm{N}$-edge along with the corresponding simulated spectra (Fig. $3 \mathrm{~b}$ ) of appropriate thickness. In the spectrum of $\mathrm{Pbl}_{2}$, a broad $\mathrm{Pb} \mathrm{N}$-edge in the form of a 'bump' can be observed at around $370 \mathrm{eV}$. A strong I M-edge at $631 \mathrm{eV}$ is also observed. The spectrum of MAl presents three edges at $\sim 285$ $\mathrm{eV}, \sim 395 \mathrm{eV}$ and $\sim 631 \mathrm{eV}$, that correspond to the $\mathrm{C}$ K-edge, N K-edge and I M-edge, respectively. In the case of Kl, the I M-edge at $\sim 631 \mathrm{eV}$ and K L-edge at $\sim 295 \mathrm{eV}$ are observed in the spectra. The spectra of $\mathrm{PbCl}_{2}$ shows a trend similar to the simulated spectrum but presents variations at $\mathrm{C}, \mathrm{N}$, and $\mathrm{O}$ edge energy positions that most likely correspond to contamination or incorrect normalization at these energy positions. In comparison to the simulated spectrum, the experimental MAI spectrum appears with an inhibited iodine M-edge. This fact is supported by energy-dispersive X-ray spectroscopy (EDX) analysis of MAI films (Figure S1 and Table S1), wherein the iodine atomic percent was observed to be small compared with those of carbon and nitrogen. An offset in the OD scale can also be observed in the case of MAI spectra compared to the simulated spectra. We speculate that this could be due to either contamination of the MAI film or radiation-related damage. This would be investigated in further studies.

In order to investigate physical and chemical phenomena in PSCs using STXM, we need to identify not only the components of the perovskite but also potential by-products from the film processing and degradation processes. For example, in the study of stability in $\mathrm{MAPbl}_{3}$, the major by-products of aged perovskite are $\mathrm{Pbl}_{2}$ and $\mathrm{PbO}{ }^{[28-32]}$ Their corresponding XAS spectra are shown in Fig. 4. We confirm that $\mathrm{Pbl}_{2}$ and $\mathrm{PbO}$ have broad $\mathrm{Pb} \mathrm{N}$-edge at $370 \mathrm{eV}$ in common. However, the I M-edge only exists in the OD spectrum of $\mathrm{Pbl}_{2}$, whereas the $\mathrm{PbO}$ spectrum is relatively flat at $370 \mathrm{eV}$. Furthermore, in the OD spectrum of $\mathrm{PbO}$, we find a prominent $\mathrm{O}$ K-edge at $543 \mathrm{eV}$. Presence of oxygen moieties can also be detected in the $\mathrm{Pbl}_{2}$ spectra, highlighting the sensitivity of these techniques to degradation by products of aging in air. These two results in Fig. 3 and Fig. 4 confirm that each component of MHPs such as MAI and $\mathrm{Pbl}_{2}$ can be detected and distinguished from other components. The absorption spectra of $\mathrm{KI}$ (pure iodine, no lead) and $\mathrm{PbCl}_{2}$ (only lead, no iodine) in Fig. 3 show that we are able to reliably attribute I and $\mathrm{Pb}$ contributions to spectra of films that contain both elements. Similarly, the absorption spectra of $\mathrm{Pbl}_{2}$ and $\mathrm{PbO}$ presented in Fig. 4 are compared to their simulated spectra of appropriate thickness to assure the reliable detection of these by-products of aged $\mathrm{MAPb}_{3}$ in soft X-ray region. The low energy anomaly observed in $\mathrm{PbO}$ in the $\mathrm{C}$ K-edge region (280-310eV) in Fig. 4 a could be due to bad normalization or beamline optics contamination.

\subsection{Characterization of $\mathrm{MAPbl}_{3}$}

In general, $\mathrm{MAPbl}_{3}$, one of MHPs, is formed by the reaction of $\mathrm{Pbl}_{2}$ and MAI. The large grain size with grain boundaries in $\mathrm{MAPbl}_{3}$ are generally considered to result in high photovoltaic performance with low recombination rate. ${ }^{[33,34]}$ The relative ratio of $\mathrm{PbI}_{2}$ to $\mathrm{MAl}$ in vacuum deposition process considerably 
affects the grain size in $\mathrm{MAPbl}_{3}$. Figure 5 illustrates the use of $\mathrm{STXM}$ to characterize $\mathrm{MAPbl}_{3}$ with different $\mathrm{Pbl}_{2}$ to MAl ratios. STXM images of $\mathrm{MAPbl}_{3}$ with $\mathrm{Pbl}_{2}$ to MAl ratio of 1:0.5, 1:1 and 1:2.5 at 662 $\mathrm{eV}$ are shown in Fig. 5a, b and c, respectively. A horizontal line profile taken from the center of the image in each case is also presented. Similar to other studies ${ }^{[35,36]}$, we observe that as the amount of MAI increases, the grain size increases. The increase in grain size can be interpreted from the line profiles and also from Fig. 5a to Fig. $5 \mathrm{c}$ by the increase in domain size (dimension of regions of similar intensity) and decrease in image "roughness". Figure $5 \mathrm{~d}$ indicates spectra of $\mathrm{MAPbl}_{3}$ with the different ratios that cover, $\mathrm{C}$ K edge, $\mathrm{N} \mathrm{K}$-edge, $\mathrm{O}$ K-edge, I M-edge and $\mathrm{Pb} \mathrm{N}$-edge energy range. The overall difference in the OD of the three spectra, especially that with the $\mathrm{Pbl}_{2}$ to MAI ratio of 1:1 arises from the difference in film thickness. In order to compare the spectra and to obtain a semi-quantitative (relative) measure of organic molecules, the integrated area under the edge-normalized carbon K-edge NEXAFS spectra (Fig. 5e), the edge-jump in nitrogen K-edge NEXAFS spectra (Fig. 5f) and the integrated area under the edge-normalized iodine M-edge NEXAFS spectra (Fig. 5e) are presented in Table 1. In each case a linear background was removed before processing. Investigations related to semi-quantitative studies using NEXAFS spectra often use edge-normalized spectra. ${ }^{\left[{ }^{37-40]}\right.}$ The integrated area under both the $\mathrm{C}$ K-edge spectra and the I M-edge spectra as well as the edge jump in N K-edge spectra (Table 1) can be clearly seen to be increasing for samples with higher MAI content.

Table 1

Integrated area under the edge-normalized C K-edge NEXAFS, the edge jump in N K-edge NEXAFS spectra and the integrated area under the edge-normalized I M-edge of various $\mathrm{MAPbl}_{3}$ samples.

\begin{tabular}{|llll|}
\hline $\begin{array}{l}\mathrm{Pbl}_{2}: \text { MAl } \\
\text { deposition ratio } \\
\text { in } \mathrm{MAPbl}_{3}\end{array}$ & $\begin{array}{l}\text { Area under the edge- } \\
\text { normalized C Kedge NEXAFS } \\
\text { spectra }\left(\mathrm{OD}^{2}\right)\end{array}$ & $\begin{array}{l}\text { Edge jump (OD) } \\
\text { in N Kedge } \\
\text { NEXAFS }\end{array}$ & $\begin{array}{l}\text { Area under the edge- } \\
\text { normalized I Kedge NEXAFS } \\
\text { spectra (OD }\end{array}$ \\
\hline $1: 0.5$ & 35 & 0.008 & 149 \\
\hline $1: 1$ & 39 & 0.058 & 153 \\
\hline $1: 2.5$ & 45 & 0.065 & 169 \\
\hline
\end{tabular}

Using STXM, we confirm that as the contents of MAI, which reacts with $\mathrm{PbI}_{2}$, increases, the "higher quality" of $\mathrm{MAPbl}_{3}$ films with larger grain size can be obtained. These results are coherent with lab-based characterization, including scanning electron microscopy (SEM) and EDX (Figure S1 and Table S1). Here we would like to point out two major differences between EDX and STXM as characterization tools. The possibility of identifying chemical states (oxidation states, chemical bonds etc.) by STXM as compared to EDX is one of the most important aspects (discussed later for this study). In addition, while EDX measurements can be considered relatively fast, it is also well known that sample damage is much more prominent in EDX as compared to STXM measurements.

Figure 6 shows XAS obtained from STXM measurements of $\mathrm{MAPbl}_{3}$ with optimized ratio $\left(\mathrm{Pbl}_{2}: \mathrm{MAI}=\right.$ 1:2.5) and two different thicknesses. The two spectra of $\mathrm{MAPbl}_{3}$ with $25 \mathrm{~nm}$ and $50 \mathrm{~nm}$ thicknesses have 
similar features with the expected difference in OD that corresponds to the difference in thickness. The clearly observed essential absorption edges (C K-edge, $\mathrm{N} \mathrm{K}$-edge, O K-edge and I M-edge) further confirms the reliability of STXM to detect thickness variations in $\mathrm{MAPbl}_{3}$ films with similar chemical composition. The sharp drop observed in the $\mathrm{C}$ K-edge region is possibly due to incorrect normalization in this region or due to carbon contamination on the beamline optics during these particular measurements. We do note that the OD does not show a perfect proportionality to thickness changes in Fig. 6 . We suspect a larger error in measurements of lower thicknesses during deposition.

Inhomogeneous morphology was observed in $\mathrm{MAPbl}_{3}$ with $1: 2.5$, and consequently a spatially resolved analysis was performed to investigate the chemical homogeneity in $\mathrm{MAPbl}_{3}$. Figure 7a shows a STXM images of $\mathrm{MAPbl}_{3}$ with the ratio of 1:2.5 at $662 \mathrm{eV}$. Figures $7 \mathrm{~b}, \mathrm{c}$, and d indicate the $\mathrm{C}, \mathrm{N}$, and I cluster maps of $\mathrm{MAPbl}_{3}$ for the same region obtained from cluster analysis performed by the software MANTIS.

${ }^{[41]}$ Figs. 7e, $f$, and $g$ are corresponding background removed XAS spectra for different clusters represented in the cluster maps. In all the cases, the spectral shape according to the clusters can be considered similar, and the variation observed arises only from the overall intensity. The intensities of spectra arising from clusters 1 and 2 are almost identical but vary in each case with the spectra of cluster 3. Considering the similarity in maps and spectra of clusters 1 and 2 , the morphological and chemical homogeneities are obvious. The pre-edge spectral features A $(286 \mathrm{eV})$ and $\mathrm{B}(\sim 287.5 \mathrm{eV})$ in C K-edge spectra (Fig. 7e) are related to $\mathrm{C} 1 \mathrm{~s} \rightarrow \pi^{\star}(\mathrm{C}=\mathrm{C})$ and $\mathrm{C} 1 \mathrm{~s} \rightarrow \pi^{\star}(\mathrm{C}=0)$ transitions respectively ${ }^{[41]}$. In this first study we speculate that these features indicate the presence of oxidation and/or degradation products due to the samples being exposed to air. The feature $\mathrm{C}(\sim 289.5 \mathrm{eV})$ and the shoulder just below it most likely arise from $\mathrm{C} 1 \mathrm{~s} \rightarrow \sigma^{\star}(\mathrm{C}-\mathrm{H}) /$ Rydberg peak ${ }^{[43]}$. Higher energy features in C K-edge $(>290 \mathrm{eV})$ and in N Kedge $(>405 \mathrm{eV})$ correspond to $\mathrm{C} 1 \mathrm{~s} \rightarrow \sigma^{*}$ and $\mathrm{N} 1 \mathrm{~s} \rightarrow \sigma^{*}$ transitions. The sensitivity of these two absorption edges along with Oxygen K-edge (not fully utilized in this study due to beamtime constraints) to chemical moieties is well known ${ }^{[44]}$ and spectral features in these edges could be utilized for identification of various functional groups in different MHP's.

The increase in OD of cluster 3 spectra indicates that while the chemical homogeneity persists on the grain, the thickness is higher. These cluster maps obtained from STXM provide spatially resolved spectra of not only the halide component but also organic components. They could be valuable in accessing chemical changes that could arise from aging or different kinds of stress.

\section{Conclusion}

In this study, we highlight the utility of STXM in the soft X-ray regime to characterize organic-inorganic MHPs. Compared with hard X-ray based characterization, soft X-ray based characterization allows investigation of X-ray absorption edges of both inorganic (especially $\mathrm{I}$ ) and organic (C and $\mathrm{N}$ ) components. 
We have presented the XAS spectra of $\mathrm{MAl}, \mathrm{Pbl}_{2}$, and $\mathrm{PbCl}_{2}$ and $\mathrm{KI}$ to examine the sensitivity of components in $\mathrm{MAPbl}_{3}$ including $\mathrm{C}, \mathrm{N}, \mathrm{Pb}$ in the soft $\mathrm{X}$-ray energy range. The measured spectra accord well with the simulated spectra except for MAl. The difference between experimental and simulation spectra in MAI is caused by the film of MAI with low concentration of iodine. The fact was supported by EDX results. In addition, XAS spectra of PbO, typical by-product formed by oxidation, were measured and found to have expected features. Through these measurements, we have demonstrated that soft X-ray absorption spectra are sensitive to various chemical components MHP's and its degradation by products.

Furthermore, taking advantage of the spatial sensitivity of $\mathrm{STXM}, \mathrm{MAPbl}_{3}$ with different deposition ratios of $\mathrm{Pbl}_{2}$ and $\mathrm{MAl}$, and the thickness of $\mathrm{MAPbl}_{3}$ film and its homogeneity were characterized in detail. We observed that the area under the edge-normalized C K-edge XAS spectra and the edge jump in N K-edge XAS spectra increases as the amount of MAI increases. In addition, we have shown that the OD is proportional to the thickness of $\mathrm{MAPbl}_{3}$ with an optimized deposition ratio of 1:2.5, which follows the Beer-Lambert law. In the chemical homogeneity test, the shapes of XAS spectra in the three different nanoscale clusters were found to be the same. Changes in film thickness due to grain formation manifests itself in the form of overall increase in intensity and edge-jump. The above-mentioned measurements reveal that STXM enables study of morphological and chemical homogeneity of MHPS, on the nanometer scale.

We anticipate that STXM measurements along with further detailed data processing that includes the analysis of the near edge "fine structure" will provide detailed chemical information of halide perovskite layer and interfaces between perovskite and charge transport layers in perovskite solar cells at the nanoscale. Furthermore, in-operando STXM using particular sample environments such as electrical bias and heating, etc., will be the next obvious steps that could lead to crucial clues for the mechanism of operational degradation of perovskite solar cells.

\section{Experimental Section/methods}

\section{Sample preparation}

All samples including $\mathrm{PbO}$ (Sigma Aldrich, 99\%), $\mathrm{PbCl}_{2}$ (Alpha Aesar, ultra-dry, 99.99\%), KI (Sigma Aldrich, 99 ), MAI (Greatcell Solar, >99.99 ), $\mathrm{Pbl}_{2}$ (Alpha Aesar, ultra-dry, 99.999. and $\mathrm{MAPbl}_{3}$ are prepared onto a $\mathrm{Si}_{3} \mathrm{~N}_{4}$ window (50 nm thicknesses, frame size of $5 \times 5 \mathrm{~mm}^{2}$, Norcada Inc., Edmonton, Canada). PbO, $\mathrm{PbCl}_{2}, \mathrm{KI}$ and MAI are prepared by the wet process. On the other hand, $\mathrm{Pbl}_{2}$ and $\mathrm{MAPbl}_{3}$ are based on the dry process. The $0.05 \mathrm{M}$ of $\mathrm{PbO}$ was dissolved in deionized water. Each $\mathrm{PbCl}_{2}$ and $\mathrm{KI}$ solution was prepared with $0.025 \mathrm{~g} \mathrm{ml}^{-1}$ concentration in N, N-Dimethylformamide (DMF, Sigma Aldrich, anhydrous, 99.8 ). MAl solution was prepared by dissolving MAl into DMF with a concentration of $0.125 \mathrm{M}$. All PbO, $\mathrm{PbCl}_{2}, \mathrm{Kl}$, and MAI films are spin-coated at $3000 \mathrm{rpm}$ in an $\mathrm{N}_{2}$ environment. $\mathrm{Pbl}_{2}$ with the thickness of 50 $\mathrm{nm}$ was prepared by vacuum deposition. $\mathrm{Pbl}_{2}$ was sublimated in a vacuum chamber at 300 , and the rate of $\mathrm{Pbl}_{2}$ was $1 \mathrm{As}^{-1}$. During the deposition, the vacuum level was $1.610^{-6} \mathrm{mbar}^{\mathrm{MAPb}} \mathrm{M}_{3}$ of $25 \mathrm{~nm}$ and 50 
$\mathrm{nm}$ were deposited by co-evaporation of $\mathrm{PbI}_{2}$ and MAl. The vacuum chamber was evacuated to the same pressure of $1.610^{-6}$ mbar. The temperature of $\mathrm{Pbl}_{2}$ and MAl were fixed at 300 and 180 . A pre-calibrated quartz crystal microbalance (QCM) sensor was used to monitor the rate of $\mathrm{Pbl}_{2}$ and MAl and the thickness of $\mathrm{MAPbl}_{3}$. The deposition rate of $\mathrm{Pbl}_{2}$ and MAl were 1 Ås ${ }^{-1}$ and $2.5 \AA \mathrm{s}^{-1}$, respectively.

STXM Measurements: STXM measurements were performed at the HERMES beamline of synchrotron SOLEIL. The details of the beamline are mentioned in Fig. 2 and elsewhere. ${ }^{[46]}$ A Fresnel zone plate of 25 nm outer zone width (Applied Nanotools Inc., Canada) was used to focus the monochromatic X-rays to a spot size of $(30 \mathrm{~nm})$. The transmitted X-rays are detected by a photomultiplier tube (PMT) after being converted to visible photons by the phosphorous screen. The sample is raster scanned across the $\mathrm{X}$-ray spot with the help of an interferometrically stabilized piezoelectric stage. The following protocol is recommended in order to perform studies in a reproducible fashion: (a) clean beamline optics is essential for investigating organic species, (b) if possible, in order to avoid contamination and degradation of the samples during transportation, a vacuum environment or isolated environment with minimum exposure to light is recommended. Spectral data presented in this communication (Figs. 3, 4 and 5) are primarily obtained from a stack of very low-resolution images measured at a range of predetermined energies. Each spectral point in these cases is obtained by integrating over each image. The energy range investigated for each sample is from 270 to $750 \mathrm{eV}$ covering C K-edge, N K-edge, 0 K-edge, I M-edge, and $\mathrm{Pb}$-edge. To avoid any mechanical drift and focusing issues due to long range travel of the zone plate, we had split the scans into four partially overlapping sections from $270-390 \mathrm{eV}, 370$ to $450 \mathrm{eV}, 430$ to $570 \mathrm{eV}$ and 550 to $750 \mathrm{eV}$. Focus corrections and beam alignment were performed between each chosen energy range. High spectral resolution ( $0.1 \mathrm{eV}$ energy step) was used near the absorption edges of $\mathrm{C}, \mathrm{N}, \mathrm{O}$, and I. In the intermediate energy ranges, an energy step of up to $5 \mathrm{eV}$ was used. Before real measurement, a radiation damage study is recommended. In order to obtain spatially resolved spectra, energy stacks were obtained over an area of $2 \times 2$ microns by raster scanning the sample with a step size of $30 \mathrm{~nm}$ with the same energy definition as described above.

\section{Declarations}

Acknowledgments

The authors would like to thank Ecole Doctorale of École Polytechnique and Synchrotron SOLEIL for their Ph.D. grants assigned to Haeyeon Jun. Philip Schulz thanks the French Agence Nationale de la Recherche for funding under the contract number ANR-17-MPGA-0012. The authors would also like to thank Synchrotron SOLEIL for providing beamtime at HERMES beamline.

Author contribution: SS, YB and HJ conceived the experiment. HJ and SS analyzed the data. HJ wrote the manuscript and all authors reviewed the manuscript. All authors participated in the experiments.

Data availability: The data generated during and/or analysed during the current study are available from the corresponding author on reasonable request. 
Additional Information: The authors declare no competing interests.

Supporting Information Supporting Information is available from the author.

\section{References}

1. D. B. Mitzi, C. A. Feild, Z. Schlesinger, R. B. Laibowitz, J. Solid State Chem. 1995, 114, 159.

2. S. D. Stranks, G. E. Eperon, G. Grancini, C. Menelaou, M. J. P. Alcocer, T. Leijtens, L. M. Herz, A. Petrozza, H. J. Snaith, 2013, 342, 341.

3. J. H. Noh, S. H. Im, J. H. Heo, T. N. Mandal, S. II Seok, Nano Lett. 2013, 13, 1764.

4. M. Saliba, J. P. Correa-Baena, M. Grätzel, A. Hagfeldt, A. Abate, Angew. Chemie - Int. Ed. 2018, 57, 2554.

5. S. F. Solari, S. Kumar, J. Jagielski, C. Shih, J. Soc. Inf. Disp. 2019, 1.

6. E. J. Yoo, M. Lyu, J. H. Yun, C. J. Kang, Y. J. Choi, L. Wang, Adv. Mater. 2015, 27, 6170.

7. F. Haque, N. T. T. Hoang, J. Ji, M. Mativenga, IEEE Electron Device Lett. 2019, 40, 1756.

8. "NREL efficiency chart," can be found under https://www.nrel.gov/pv/cell-efficiency.html, n.d.

9. H. J. Jung, D. Kim, S. Kim, J. Park, V. P. Dravid, B. Shin, Adv. Mater. 2018, 30, 1802769.

10. A. Uddin, M. B. Upama, H. Yi, L. Duan, Coatings 2019, 9, 65.

11. A. F. Akbulatov, L. A. Frolova, M. P. Griffin, I. R. Gearba, A. Dolocan, D. A. Vanden Bout, S. Tsarev, E. A. Katz, A. F. Shestakov, K. J. Stevenson, P. A. Troshin, Adv. Energy Mater. 2017, 7, 1.

12. P. Sedigh Rahimabadi, M. Khodaei, K. R. Koswattage, X-Ray Spectrom. 2020, 49, 348.

13. G. W. P. Adhyaksa, S. Brittman, H. Āboliṇš, A. Lof, X. Li, J. D. Keelor, Y. Luo, T. Duevski, R. M. A. Heeren, S. R. Ellis, D. P. Fenning, E. C. Garnett, Adv. Mater. 2018, 30, 1804792.

14. X. Li, Y. Luo, M. V. Holt, Z. Cai, D. P. Fenning, Chem. Mater. 2019, 31, 2778.

15. Y. Luo, S. Gamliel, S. Nijem, S. Aharon, M. Holt, B. Stripe, V. Rose, M. I. Bertoni, L. Etgar, D. P. Fenning, Chem. Mater. 2016, 28, 6536.

16. M. E. Stuckelberger, T. Nietzold, B. M. West, Y. Luo, X. Li, J. Werner, B. Niesen, C. Ballif, V. Rose, D. P. Fenning, M. I. Bertoni, J. Phys. Chem. C 2020, 124, 17949.

17. B. Philippe, M. Saliba, J. P. Correa-Baena, U. B. Cappel, S. H. Turren-Cruz, M. Grätzel, A. Hagfeldt, H. Rensmo, Chem. Mater. 2017, 29, 3589.

18. T. Sekimoto, T. Matsui, T. Nishihara, R. Uchida, T. Sekiguchi, T. Negami, ACS Appl. Energy Mater. 2019, 2, 5039 .

19. F. G. Santomauro, J. Grilj, L. Mewes, G. Nedelcu, S. Yakunin, T. Rossi, G. Capano, A. Al Haddad, J. Budarz, D. Kinschel, D. S. Ferreira, G. Rossi, M. G. Tovar, D. Grolimund, V. Samson, M. Nachtegaal, G. Smolentsev, M. V. Kovalenko, M. Chergui, Struct. Dyn. 2017, 4, 044002.

20. C. Vorwerk, C. Hartmann, C. Cocchi, G. Sadoughi, S. N. Habisreutinger, R. Félix, R. G. Wilks, H. J. Snaith, M. Bär, C. Draxl, J. Phys. Chem. Lett. 2018, 9, 1852. 
21. M. Kodur, R. E. Kumar, Y. Luo, D. N. Cakan, X. Li, M. Stuckelberger, D. P. Fenning, Adv. Energy Mater. 2020, $10,1$.

22. L. M. Wheeler, E. M. Sanehira, A. R. Marshall, P. Schulz, M. Suri, N. C. Anderson, J. A. Christians, D. Nordlund, D. Sokaras, T. Kroll, S. P. Harvey, J. J. Berry, L. Y. Lin, J. M. Luther, J. Am. Chem. Soc. 2018, $140,10504$.

23. H. Lee, S. Gaiaschi, P. Chapon, D. Tondelier, J. Boureé, Y. Bonnassieux, V. Derycke, B. Geffroy, 2019, $123,17728$.

24. B. L. Henke, E. M. Gullikson, J. C. Davis, At. Data Nucl. Data Tables 1993, 54, 181.

25. H. Hansen, Ann. Phys. (N. Y). 1939, 427, 524.

26. F. J. Comes, R. Haensel, U. Nielsen, W. H. E. Schwarz, J. Chem. Phys. 1973, 58, 516.

27. aXis2000, available freely at http://unicorn.chemistry.mcmaster.ca/axis/aXis2000-download.html 28. G. Niu, W. Li, F. Meng, L. Wang, H. Dong, Y. Qiu, J. Mater. Chem. A 2014, 2, 705.

29. Y. H. Kye, C. J. Yu, U. G. Jong, Y. Chen, A. Walsh, J. Phys. Chem. Lett. 2018, 9, 2196.

30. J. Yang, Z. Yuan, X. Liu, S. Braun, Y. Li, J. Tang, F. Gao, C. Duan, M. Fahlman, Q. Bao, ACS Appl. Mater. Interfaces 2018, 10, 16225.

31. Y. Ouyang, Y. Li, P. Zhu, Q. Li, Y. Gao, J. Tong, L. Shi, Q. Zhou, C. Ling, Q. Chen, Z. Deng, H. Tan, W. Deng, J. Wang, J. Mater. Chem. A 2019, 7, 2275.

32. Q. Sun, P. Fassl, D. Becker-Koch, A. Bausch, B. Rivkin, S. Bai, P. E. Hopkinson, H. J. Snaith, Y. Vaynzof, Adv. Energy Mater. 2017, 7, 1700977.

33. R. Long, J. Liu, O. V. Prezhdo, J. Am. Chem. Soc. 2016, 138, 3884.

34. M. Nukunudompanich, G. Budiutama, K. Suzuki, K. Hasegawa, M. Ihara, CrystEngComm 2020, 22, 2718.

35. H.-S. Yun, Y. H. Jang, D.-K. Lee, Curr. Photovolt. Res. 2018, 6, 12.

36. S. Sanders, D. Stummler, J. D. Gerber, J. H. Seidel, G. Simkus, M. Heuken, A. Vescan, H. Kalisch, MRS Adv. 2020, 5, 385.

37. S. Swaraj, U. Oran, A. Lippitz, R. D. Schulze, J. F. Friedrich, W. E. S. Unger, Plasma Process. Polym. $2004,1,134$.

38. S. Swaraj, U. Oran, A. Lippitz, J. F. Friedrich, W. E. S. Unger, Plasma Process. Polym. 2005, 2, 572.

39. S. Swaraj, U. Oran, A. Lippitz, J. F. Friedrich, W. E. S. Unger, Plasma Process. Polym. 2007, 4, 784.

40. S. Swaraj, U. Oran, A. Lippitz, W. E. S. Unger, Plasma Process. Polym. 2008, 5, 92.

41. M. Lerotic, R. Mak, S. Wirick, F. Meirer, C. Jacobsen, J. Synchrotron Radiat. 2014, 21, 1206.

42. Urquhart SG, Ade H. J Phys. Chem. B 2002,106:8531.

43. S. Swaraj, H. Ade, J. Electr. Spectr. Rel. Phen. 2013, 191, 60

44. R. Belkhou, S. Stanescu, S. Swaraj, A. Besson, M. Ledoux, M. Hajlaoui, D. Dalle, J. Synchrotron Radiat. 2015, 22, 968. 


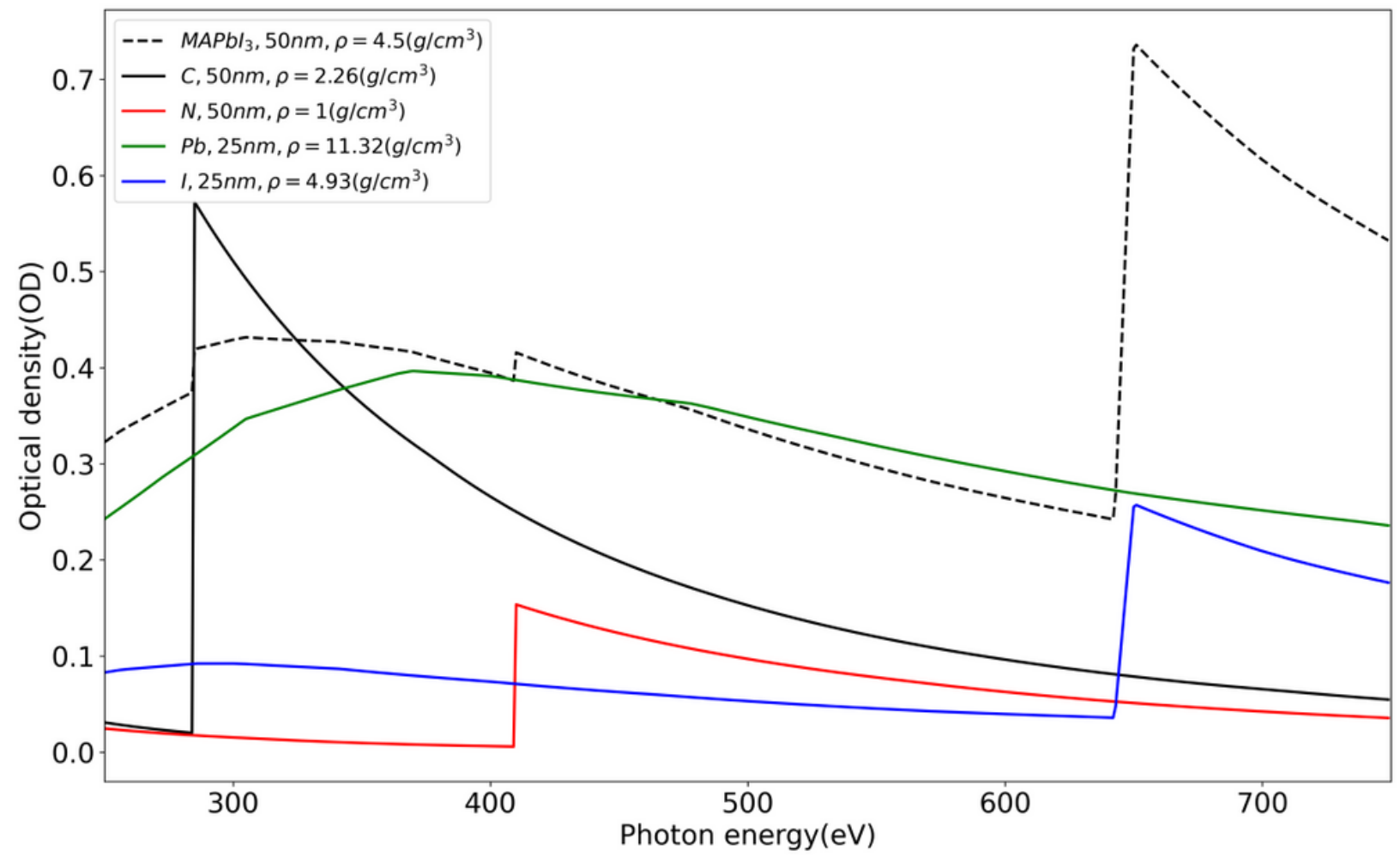

Figure 1

Simulations for X-ray absorption spectrum of $\mathrm{MAPbl}_{3}$ (top) and the different perovskite elements including $\mathrm{C}, \mathrm{N}, \mathrm{Pb}$ and I (bottom) in soft X-ray region using aXis2000 [27]. 
(a)

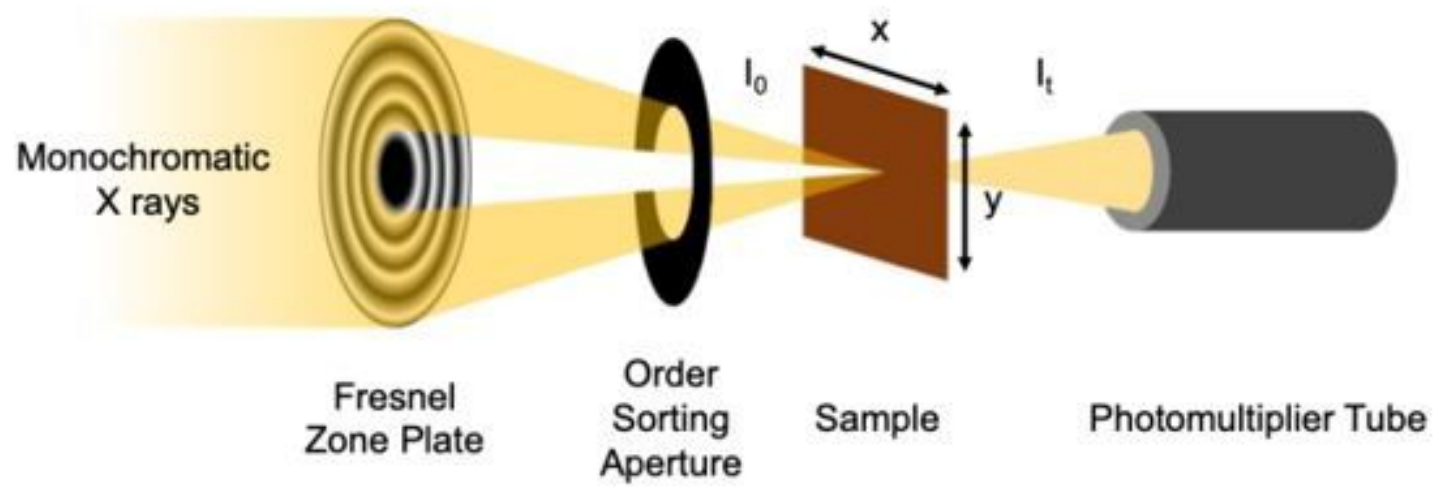

(b)

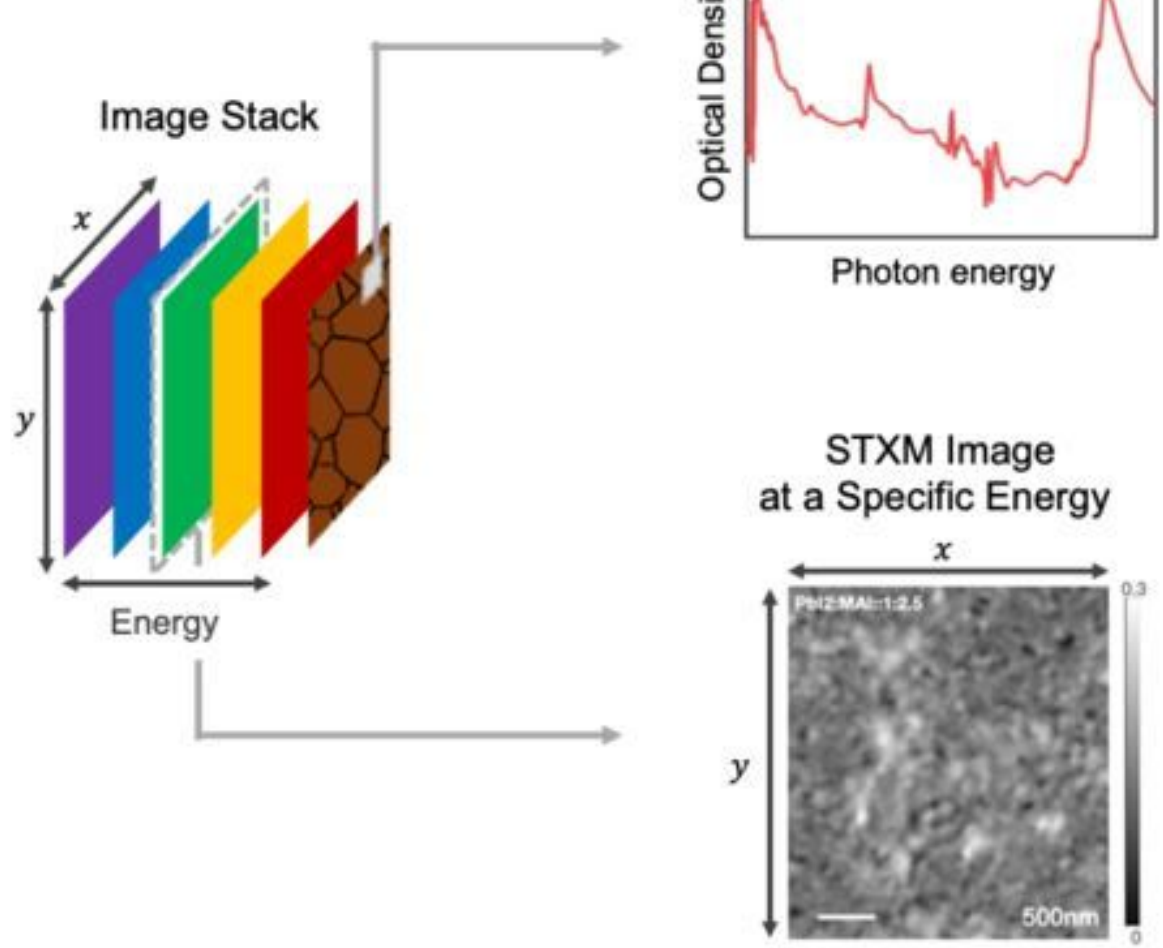

Figure 2

a) Experimental set-up for scanning transmission X-ray microscopy (STXM). b) Illustration of data acquired by STXM, including spatially resolved spectra (spectrum in a particular pixel) and STXM image at a specific energy. 


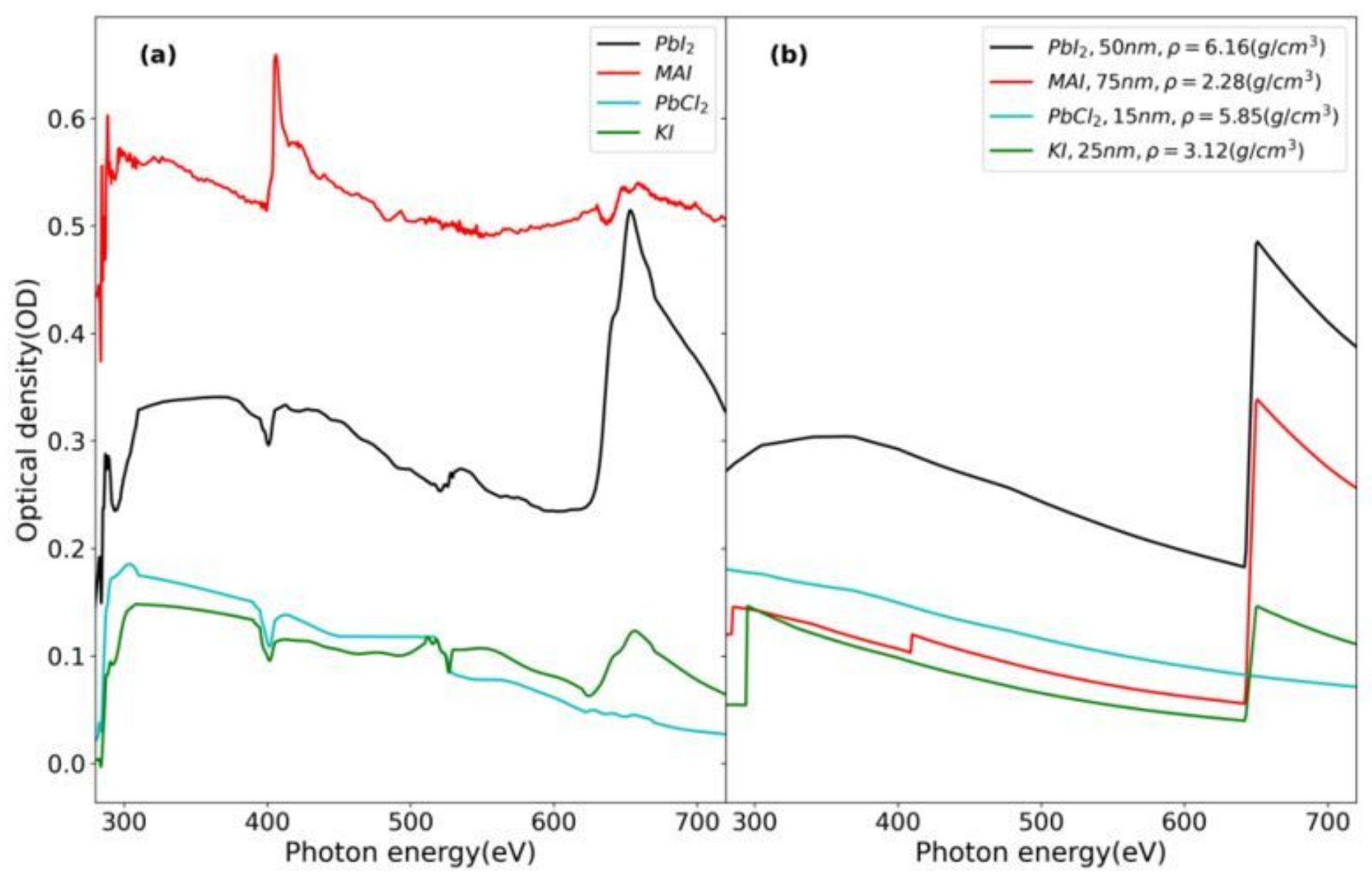

Figure 3

a) Experimental XAS spectra and b) Simulated spectra ${ }^{[27]}$ of $\mathrm{Pbl}_{2}, \mathrm{MAl}, \mathrm{PbCl}_{2}$ and $\mathrm{KI}$ in the range of 280$720 \mathrm{eV}$. Simulation parameters are mentioned in legend of $(b)$ 


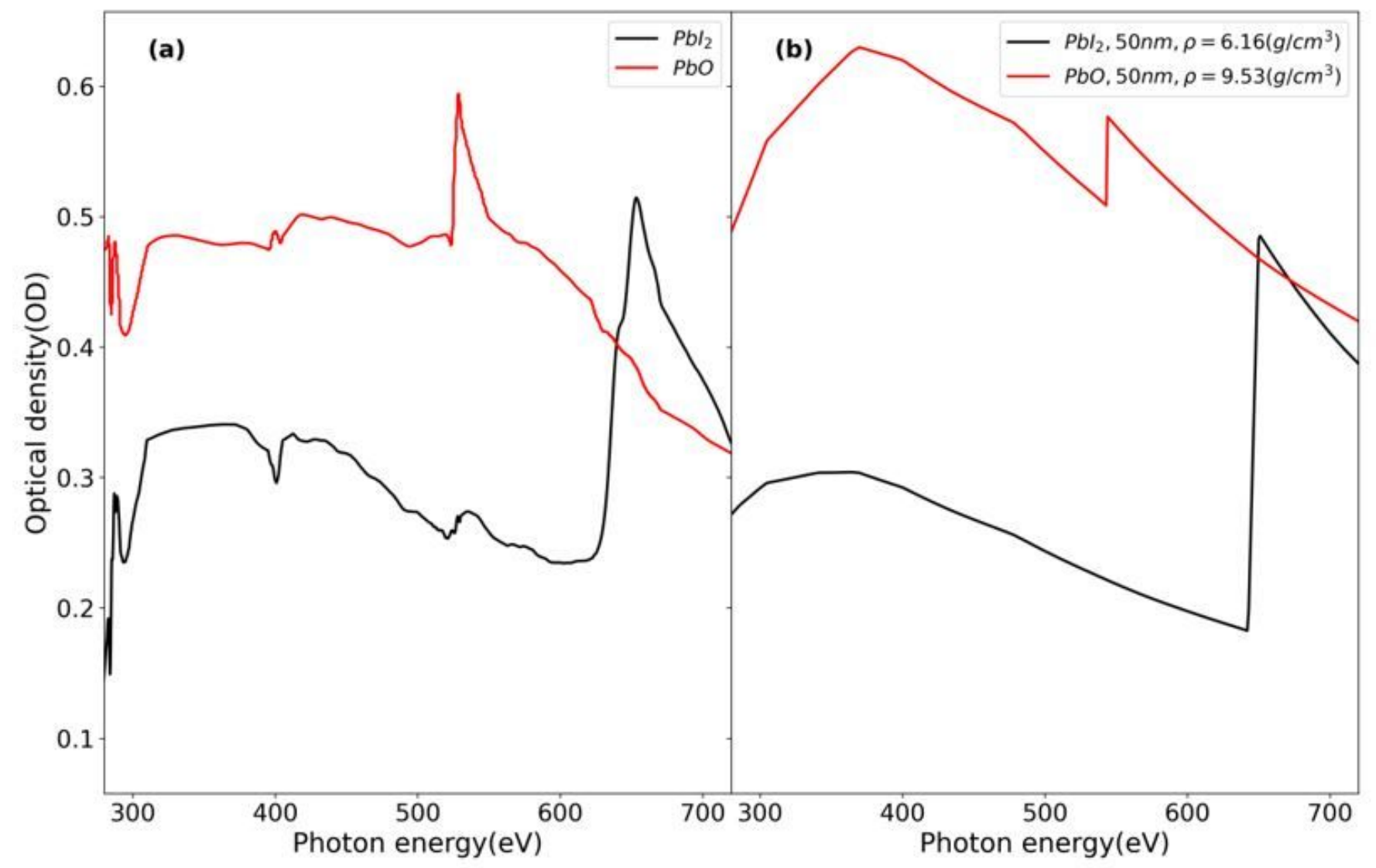

Figure 4

a) Experimental NEXAFS spectra and b) Simulated spectra ${ }^{[27]}$ of $\mathrm{Pbl}_{2}$, and $\mathrm{PbO}$ in the range of $280-720$ $\mathrm{eV}$. Simulation parameters are mentioned in legend of (b) 

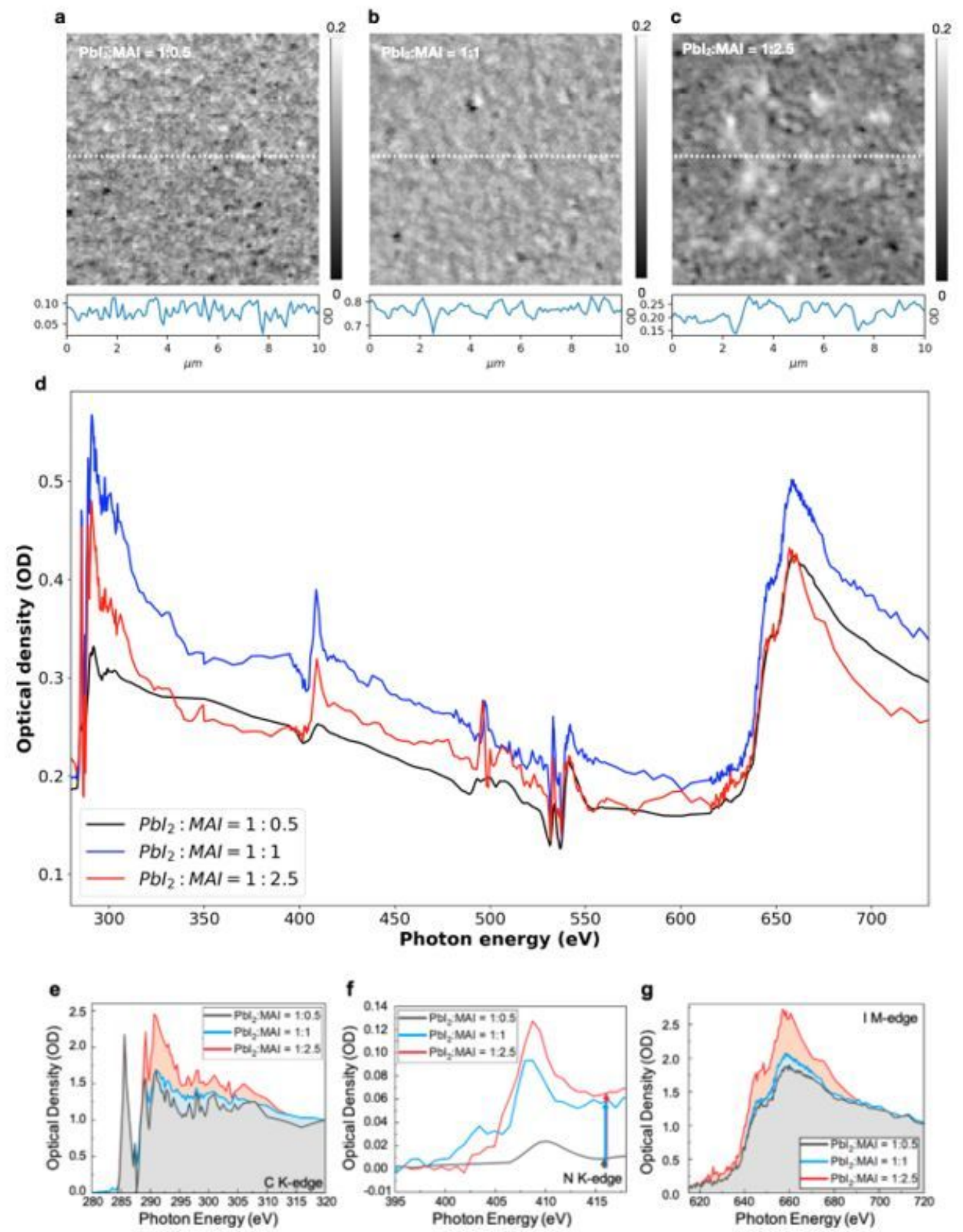

\section{Figure 5}

STXM images and spectra of $\mathrm{MAPbl}_{3}$ with different $\mathrm{Pbl}_{2}$ to MAl ratios. STXM images and line profile (taken from white dotted line in the image) at $662 \mathrm{eV}$ with the ratio of a) 1:0.5 b) 1:1 c) 1:2.5, d) NEXAFS spectra in the entire energy region ( $280-730 \mathrm{eV})$, e) edge normalized C K-edge, f) N K-edge, and g) I Medge region of $\mathrm{MAPbl}_{3}$ with different $\mathrm{Pbl}_{2}$ to MAl ratios. The shaded regions in e) and g) indicate the integrated C K-edge spectra and I M-edge spectra, and the arrows in f) represents the N K-edge jumps 


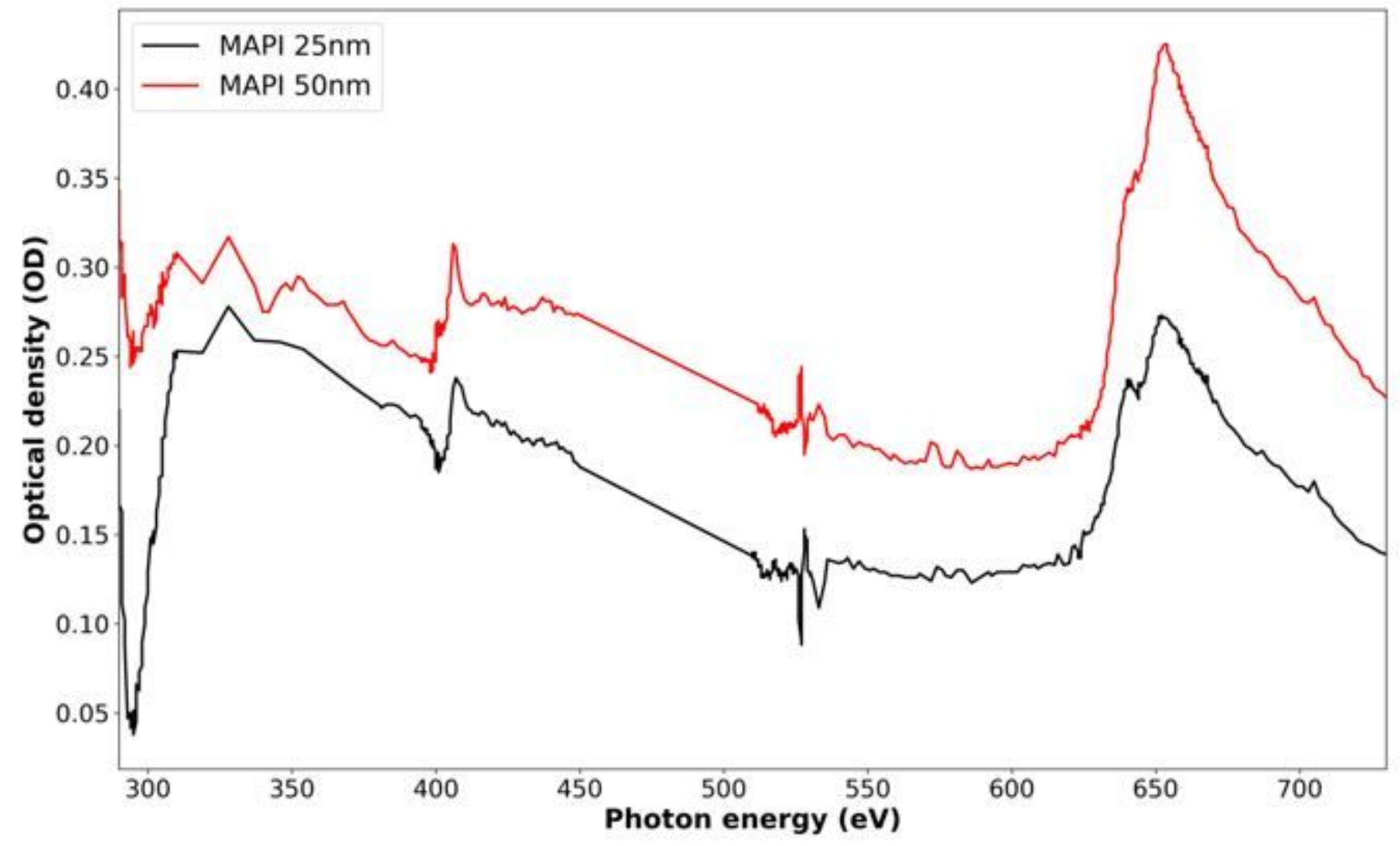

Figure 6

XAS spectra of $\mathrm{MAPbl}_{3}$ with optimized $\mathrm{Pbl}_{2}$ to MAl ratio (1:2.5) and two different thicknesses of $25 \mathrm{~nm}$ and $50 \mathrm{~nm}$. 


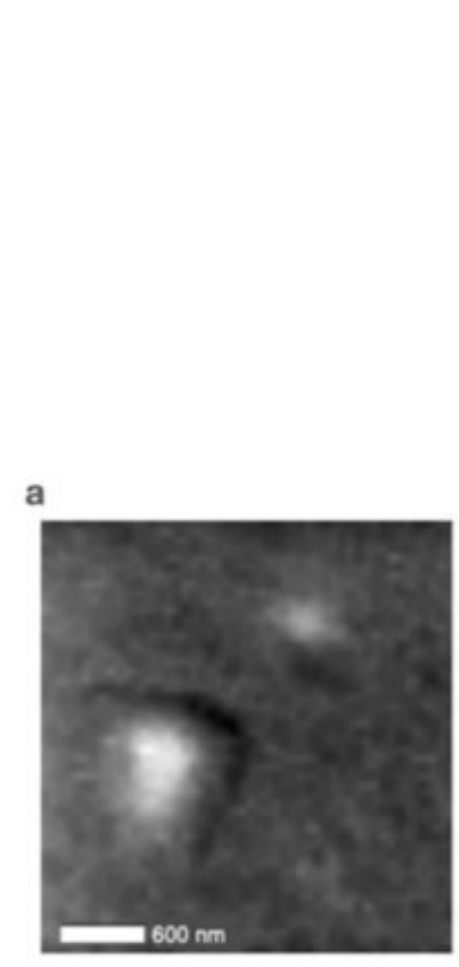

b

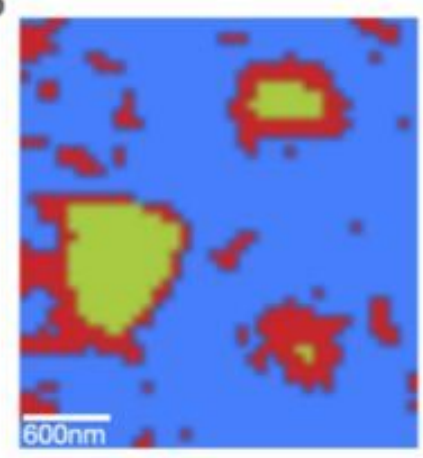

c

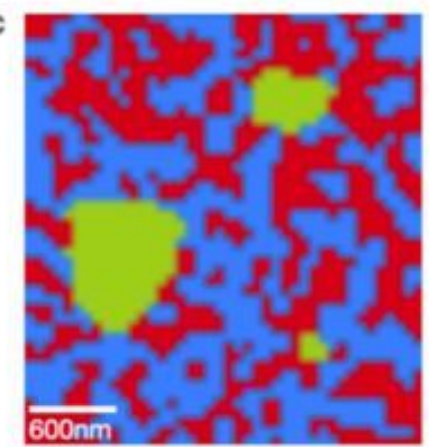

d

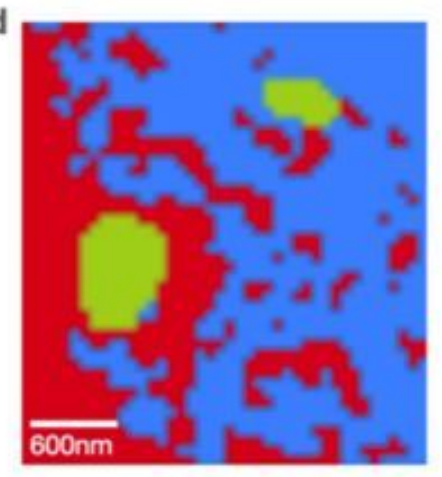

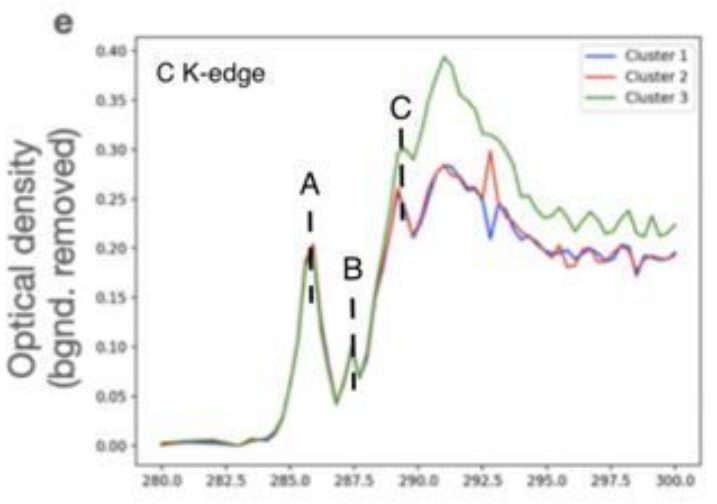
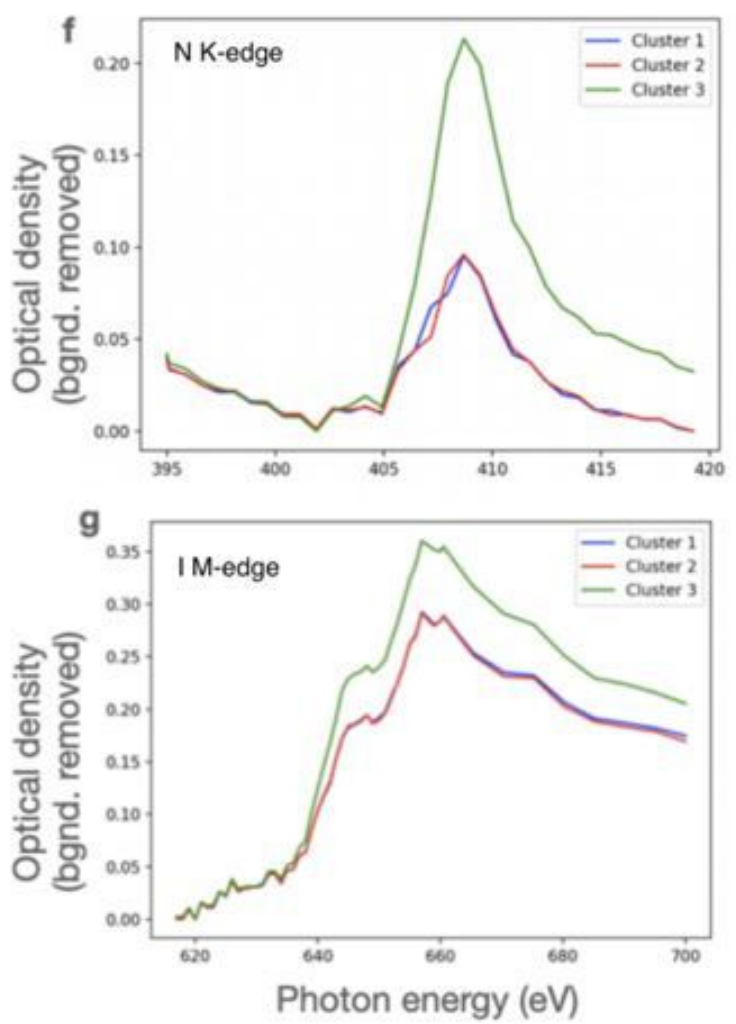

Figure 7

a) STXM images at $662 \mathrm{eV}$ of $\mathrm{MAPbl}_{3}$ with $\mathrm{Pbl}_{2}$ to MAl ratio of 1:2.5. Cluster images of $\mathrm{MAPbl}_{3}$ in the b) C K-edge, c) N K-edge, and d) I M-edge region, Corresponding average cluster XAS spectra of cluster analysis in the e) C K-edge, f) N K-edge, and g) I M-edge region (Color of each spectrum corresponds to color of each cluster on the map).

\section{Supplementary Files}

This is a list of supplementary files associated with this preprint. Click to download.

- SIHaeyeonJuncorrectedrevised.docx 